\title{
Vision impairment as the first symptom of encephalomyelitis in a paediatric patient
}

\section{Aleksandra Świerczyńska, Małgorzata Woś}

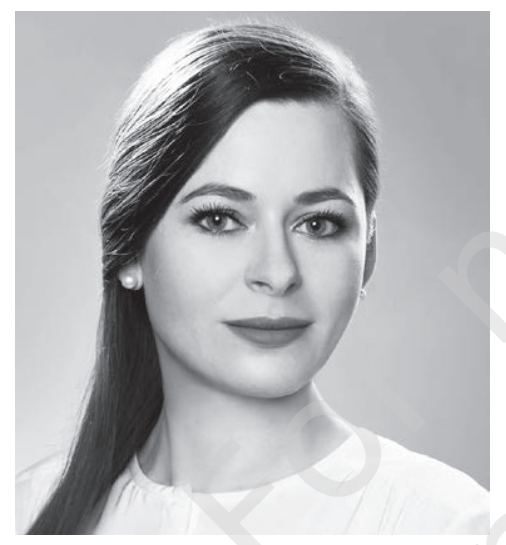

\section{H I G H L I G H T S}

Vision impairment may be the first symptom of a rare neurological disorder, so the diagnosis of each case should be done based on the overall clinical picture.

\section{ABSTRACT}

An 8-year-old boy was admitted to the Department of Ophthalmology (Stefan Żeromski Hospital, Cracow, Poland) due to vision impairment with an accompanying decrease in visual acuity and bilateral optic disc oedema. As a result of interdisciplinary diagnostics, acute disseminated encephalomyelitis, probably triggered by a preceding infection, was diagnosed.

Key words: vision impairment, encephalomyelitis, ADEM, demyelination 


\section{INTRODUCTION}

Visual impairment is one of the most common reasons for patients to seek an urgent ophthalmological consultation [1]. However, it often causes numerous diagnostic problems, which stems both from the patient's difficulty in describing their subjective complaints and from the occurrence of diseases in which ophthalmic symptoms are only a manifestation of a disease of, e.g., of neurological or internistic origin.

Ophthalmologic diseases that cause the decrease of visual acuity (VA) may affect each section of the eyeball, and the most frequently responsible are inflammatory conditions, vascular disorders within the retinal vessels and pathologies of the retina, cornea, bleeding into the vitreous body, inflammation of the optic nerve or trauma [1]. Less common conditions such as neuropathies (e.g., demyelinating, toxic, post-traumatic), migraine, and even anatomical defects (e.g., cervical rib syndrome) should also be remembered [1]. This is important especially in pediatric patients, because their clinical history related to subjective complaints may be difficult or unclear.

\section{CASE REPORT}

An 8-year-old boy presented for an ophthalmology consultation during his stay in the Hospital Emergency Department because of decreased VA in the right eye that had appeared several hours earlier. On admission, the complaints were not accompanied by other ophthalmic or general symptoms.

The mother of the boy reported visual disturbances in the left eye of similar character as on the day of consultation about 2 months earlier (deterioration of vision - the complaints resolved spontaneously). Approximately 1 month earlier the child's mother noticed severe headaches with body temperature above $38^{\circ} \mathrm{C}$ accompanied by vomiting. At that time an attempt of ambulatory treatment was undertaken (azithromycin), but as the complaints did not resolve, the boy was admitted to the Pediatric Ward of the district hospital, where diagnostics was performed, among others for Lyme disease, presence of parasites in the stool, as well as brain MRI, which did not show any abnormalities. After neurological examination migraine without aura was diagnosed and the child was referred for further outpatient treatment. Moreover, in the week preceding the visit in the local Emergency Department, the boy developed a fever of $38.6^{\circ} \mathrm{C}$, therefore amoxicillin and naproxen were added to the treatment.

The boy denied any ocular or head trauma. Ophthalmologic examination stated:
BCVA OD 0.2 s.c.
Ta OD $20 \mathrm{mmHg}$
BCVA OS 0.2 s.c.
Ta OS $21 \mathrm{mmHg}$

Ocular correction based on the results of the refraction examination did not affect VA. Direct and indirect pupillary reaction to light was appropriate, the mobility of eyeballs was proper, the convergence was preserved, the boy did not report any symptoms of eye twitching or pain on movements, the recognition of colors and sense of contrast was correct, the approximate field of vision was preserved, the cover test was negative for distance and near.

No abnormalities were found in the anterior segment of both eyes, but bilateral fundus examination showed significant elevation of the optic nerve discs with blurring of their borders, which was further confirmed by OCT examination of the discs (fig. 1). Optical coherence tomography of the maculae showed no abnormalities, as did ultrasound examination (fig. 2).

\section{FIGURE 1}

Fundus photographs at the time of admission.

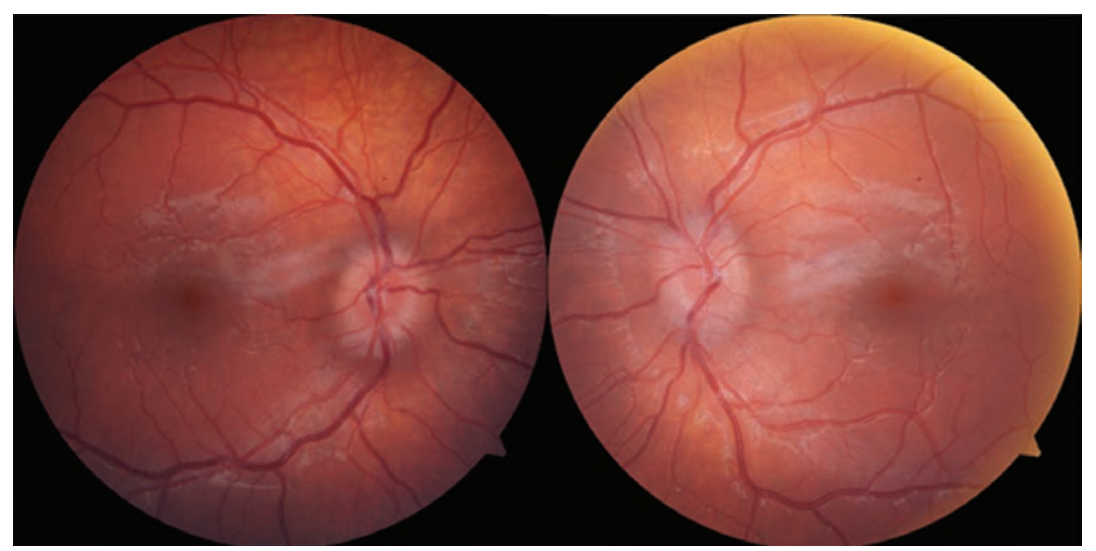




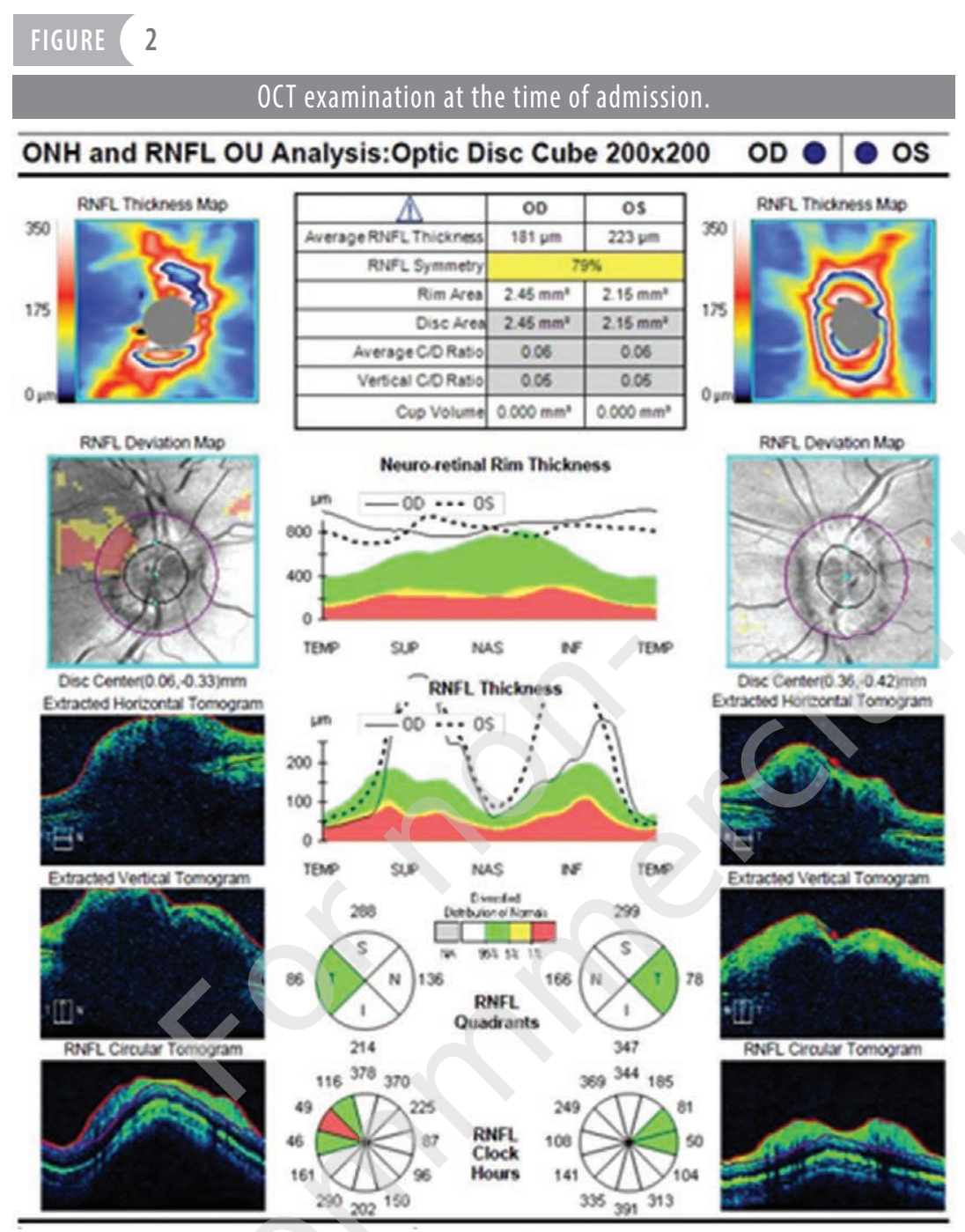

Due to a normal result of a recent brain MRI provided by the mother, a CT scan was abandoned and the boy was consulted with a pediatric infectious disease physician - however, the consultation did not provide diagnostically relevant information.

Because of the abnormalities found in the examination, it was recommended to extend the diagnostic process within the stay in the Department of Ophthalmology. The boy's mother did not agree to hospitalization due to the holiday season; therefore, the patient was scheduled to be admitted to the Department of Ophthalmology three days later.

On the day of admission, ophthalmologic examination revealed the following changes in VA:

$\begin{array}{ll}\text { BCVA OD } 0.1 \text { s.c. } & \text { Sn OD } 3.0 \text { s.c. } \\ \text { BCVA OS } 0.4 \text { s.c. } & \text { Sn OS } 0.75 \text { s.c. }\end{array}$

Additionally, there was a decrease in red color saturation in the right eye and irregular white-spot visual field loss in the Amsler test bilaterally. Body temperature remained normal. In addition, the mother observed the boy's gait instability, decreased appetite, and weakness over the past 2 days. Ac- cording to her report, 3 months earlier the boy and his family had experienced a flu-like infection with body temperature above $40^{\circ} \mathrm{C}$. Other relevant history information included a history of CMV infection, mononucleosis, and prematurity (born at $34 \mathrm{Hbd}$ ). The child's development to that time was normal.

After the patient was admitted to the Department, local treatment was initiated (antibiotic and steroid therapy), laboratory and imaging examinations, including those for zoonotic diseases, as well as specialist consultations were scheduled. On the same day, during the duty hours, while administering medications, the boy developed collapse without loss of consciousness with severe weakness, slurred speech and balance disorders. An in-depth medical history from the mother revealed recent episodes of urinary and fecal retention, as well as behavioral and mood disturbances such as severe anxiety, fear, periods of apathy or agitation, and aggression towards parents.

It was decided to refer the boy for urgent pediatric consultation, which showed only slightly elevated body temperature $\left(37.4^{\circ} \mathrm{C}\right)$ and heart rate $(130 \mathrm{bpm})$. Meningeal symp- 
toms and the Romberg test remained negative. The results showed ASO $3227 \mathrm{IU} / \mathrm{ml}$, leukocytosis $9.4 \times 10^{9} / 1$ with normal blood cells, CRP $1.1 \mathrm{mg} / \mathrm{l}$, adnexal densities in chest $\mathrm{X}$-ray, other parameters did not show any abnormalities. Ear, nose, and throat consultation did not bring information about inflammatory foci or other abnormalities. Thus, the decision was made to urgently transfer the boy to the Department of Pediatrics and Child Neurology at another hospital. On admission emotional disturbances, global muscle tone reduction, marked meningeal symptoms, trace of tremor and dysmetria, clumsy and unsteady gait were found. Family history additionally revealed the presence of multiple sclerosis (MS) in two sisters of the patient's grandmother. During hospitalization the diagnostics was expanded to included CSF examination, repeat MRI of the brain, cervical and thoracic spine, which this time showed demyelinating lesions in the thalamus and involvement of the right optic nerve, as well as visual evoked potentials examination, where the recordings from stimulation of the right and left eye showed prolonged latency of the P100 wave, characteristic of inflammatory diseases of the optic nerve [1]. The awake EEG, ECG and abdominal ultrasound were within normal limits. A complete diagnostic PCR for bacterial and viral disorders from both blood and cerebrospinal fluid was also performed and negative results were obtained (infections excluded: Haemophilus influenzae, Neiseria meningitidis, Streptococcus pneumoniae, Enterovirus, Epstein-Barr virus, Human Adenovirus, Herpes Simplex virus 1\&2, Human Cytomegalovirus, Varicella Zoster virus, Human Herpes virus 6\&7, Human Parechovirus, Human Parvovirus B19). The levels of IgM and IgG antibodies against HSV were negative in the cerebrospinal fluid. C3 and C4 complement levels remained normal. Re-diagnosis for Lyme disease was also negative, as were cytomegalovirus and Mycoplasma pneumoniae infections. Bacterial cultures of blood and cerebrospinal fluid for aerobic bacteria were negative.

Cerebrospinal fluid examination revealed elevated cytosis $(100 \mathrm{H} / \mu \mathrm{l})$, protein levels $(0.52 \mathrm{~g} / \mathrm{l})$, and decreased glucose ( $2.6 \mathrm{mmol} / \mathrm{l})$.

Based on the above diagnostics, acute disseminated encephalomyelitis (ADEM) was recognized.

As a result of in-patient and out-patient treatment, which included antibiotic and steroid therapy, the symptoms disappeared and full VA was restored. A follow-up brain MRI performed at the end of the 3-week hospitalization showed partial regression of the lesions and no new lesions. An ophthalmologic examination performed 4 weeks after hospitalization showed VA of 0.8 s.c. in both eyes and regression of optic nerve disc edema. Visual field examination showed no abnormalities. During the follow-up 8 months after hospitalization, bilateral full VA for distance and near was found without any correction with glasses, and the oedema of the optic nerve discs resolved. At present, the patient is under permanent supervision of the Neurological Outpatient Clinic and Ophthalmological Outpatient Clinic.

\section{DISCUSSION}

Acute disseminated encephalomyelitis (ADEM) is a rare disease of the central nervous system (1 in 125,000250,000 cases per year [2]) involving autoimmune demyelinating processes. It usually presents around the age of 7 years, slightly more often in boys, usually in spring or winter, and is often (50-75\% [2]), although not always, associated with a preceding viral or, less commonly, bacterial infection [2-5] (usually group A streptococcus) [6]. The time between infection and the development of ADEM symptoms usually ranges from a few days to about 2 months, but up to $25 \%$ of patients fail to have a preceding infection [2]. Very rare triggers for ADEM mentioned in the literature may be malignancy (as a paraneoplastic syndrome), allogeneic bone marrow transplantation, autoimmune hemolytic anemia, systemic lupus erythematosus, or active immunization $[2,4,5,7]$. Lesions can affect any location in the central nervous system, but most commonly the white matter motor pathways, optic nerves, and spinal cord [8].

The majority of patients with exacerbation of the disease show behavioral and consciousness disturbances, fever, headache, weakness, clumsiness, vomiting, meningeal symptoms, and in some patients also optic neuritis (7-23\% of patients [5]), oculomotor disorders, urinary retention, ataxia, aphasia, dysarthria, seizures or even signs of increased intracranial pressure $[2,4,5]$.

The diagnosis of ADEM is mainly based on MRI detecting relatively characteristic demyelinating lesions (single or diffuse, asymmetrical patches with indistinct borders, usually occurring bilaterally) [2]. However, it should be remembered that (as in the case of our patient) they are not necessarily visible at an early stage of the disease, and in a small proportion of patients they appear only after many weeks or not at all [2]. Moreover, the MRI examination allows for the assessment of the disease stage and is helpful in differentiating ADEM from MS [6]. A characteristic feature of ADEM flares is their gradual resolution and, more importantly, the absence of new plaques on follow-up examinations [5]. It is also important to exclude bacterial and viral neuroinfections and inflammatory diseases before making a definitive diagnosis. For this purpose, it is necessary to perform tests from cerebrospinal fluid and blood of the patient $[2,7,9]$. EEG recordings can reveal disturbed sleep pattern and slowed baseline brain function [2]. When optic nerve disorders are present, evaluation of visual evoked potentials may also be helpful $[4,8]$. All the methods men- 
tioned above were used in the diagnostic process of the discussed patient.

The treatment based on clinical data is mainly high-dose steroid therapy, immunoglobulins and plasmapheresis (PLEX), which are effective in the majority of patients $[2,4,5]$. Further management depends on the patient's condition and may include administration of antibiotics, antivirals (acyclovir), antipyretics, anticonvulsants, in rare cases cyclophosphamide, and even intensive care unit (ICU) care $[2,3,5]$. Although the prognosis is usually good, with complete remission occurring by about 1 month after disease onset, in a notable proportion of cases, the disease leaves neurological deficits or cognitive impairment of at least a small degree $[2-5,10]$. Today, the mortality rate of ADEM is about 5\% and concerns mainly its rare, hyperacute forms with inflammatory-hemorrhagic course (mortality in this group is still up to 50\%). A decrease in mortality has been achieved, among others, by the introduction of widespread measles vaccination, which was previously a significant risk factor of the disease $[4,5]$.

\section{ACUTE DISSEMINATED ENCEPHALOMYELITIS AND MULTIPLE SCLEROSIS}

Acute disseminated encephalomyelitis and multiple sclerosis (MS) are sometimes difficult to distinguish, especially in adults, but in clinical practice it is necessary because the treatment and prognosis of the two cases differ significantly [2]. Important clues in distinguishing the disorders are not only the appearance of the plaques on MRI, but also the presence of preceding infection, fever (more common in ADEM), disturbances of consciousness and emotions (more common in ADEM), and a monosymptomatic course (characteristic of MS, e.g., optic neuritis only) [2]. Given the numerous similarities, some researchers suggest that there is a continuity in the clinical picture between ADEM and MS, which could also include idiopathic optic neuritis, transverse myelitis or Devic's syndrome [8]. Due to numerous similarities between the above mentioned entities, differentiating between them can be challenging, especially in the case of multiphasic ADEM, and should be left to specialists in pediatrics and neurology [4]. At the current stage of knowledge, it cannot be excluded with certainty that these conditions are different manifestations of the same disease. The risk of ADEM progressing to MS is 25-29\% [3, 8] (in the adult population as high as $35 \%$ [5]), therefore constant control of the patient in the neurological and ophthalmological clinics is extremely important. This is particularly relevant for patients who, similarly to our patient, have a positive family history of demyelinating diseases.

From the perspective of ophthalmic practice, an important feature indicating the possibility of ADEM is that optic nerve lesions with bilateral manifestations are more frequent than in MS, especially in pediatric patients $[2,4]$. Symptoms do not have to begin simultaneously in both eyes; however, an evoked potential testing in the unoccupied eye may demonstrate changes even before the clinical onset of symptoms [8]. Other less common ocular symptoms include oculomotor dysfunction and nystagmus [2]. Nevertheless, these are uncharacteristic symptoms and only by considering the entire clinical picture, including detailed history, can the diagnostic process be directed in the right path.

\section{CONCLUSIONS}

In the retrospective evaluation of the present case, numerous discrete symptoms suggesting the possibility of ADEM already at earlier stages of treatment can be seen. However, at the time of the disease they were so uncharacteristic that only the coincidence of the complaints and a targeted interview allowed obtaining from the mother the information necessary to take diagnostic steps in the right direction.

Visual disturbances are a common reason for patients to see an ophthalmologist. Although the cause of most of them is obvious and easy to find in a standard ophthalmologic examination, in a small group of patients vision disorders may be the first symptom of other systemic disorders or diseases from bordering on different branches of medicine. The above case illustrates an exceptionally vivid manner how important is an in-depth medical history and quick diagnostics and treatment in the diagnostic process of vision disorders. A holistic view of the patient, going beyond the framework of a typical ophthalmologic examination, may help us see in our everyday practice patients with rare diseases, who despite often discrete or uncharacteristic symptoms require immediate help.

Figures: from the author's own materials.

\section{CORRESPONDENCE}

\section{Aleksandra Świerczyńska, MD}

Department of Ophthalmology with the Children's

Ophthalmology Subdivision, Stefan Żeromski Hospital

31-913 Cracow, Osiedle Na Skarpie 66

e-mail: aleksandra.swierczynska7@gmail.com

\section{ORCID}

Aleksandra Świerczyńska - ID - http://orcid.org/0000-0002-2353-8079 Małgorzata Woś - ID - http://orcid.org/0000-0003-3184-9958 


\section{References}

1. Figurska M, Niedzielski M. Nagłe zaniewidzenie - do okulisty, neurologa czy internisty? Okulistyka po Dyplomie. 2017; 6. https://podyplomie.pl/okulistyka/29366,nagle-zaniewidzenie-do-okulisty-neurologa-czy-internisty (access: 17.05.2021).

2. Anilkumar AC, Foris LA, Tadi P. Acute Disseminated Encephalomyelitis. [Updated 2021 Jan 31]. In: StatPearls [Internet]. Treasure Island (FL): StatPearls Publishing; 2021.

3. Losy J, Selmaj K. Ostre rozsiane zapalenie mózgu i rdzenia (ADEM). Neurologia Praktyczna. https://neurologia-praktyczna.pl/a1323/ Ostre-rozsiane-zapalenie-mozgu-i-rdzenia--ADEM-.html (access: 17.05.2021).

4. Jamroz-Wiśniewska A. Ostre rozlane zapalenie mózgu i rdzenia kręgowego. Neurologia po Dyplomie. https://podyplomie.pl/wiedza/ neurologia/190,ostre-rozlane-zapalenie-mozgu-i-rdzenia-kregowego (access: 17.05.2021).

5. Javed A, Khan O. Acute disseminated encephalomyelitis. Handb Clin Neurol. 2014; 123: 705-17. https://doi.org/10.1016/B978-0-44453488-0.00035-3.

6. Kaciński M. Choroby układu nerwowego. In: Kawalec W, Grenda R, Ziółkowska H. Pediatria. Wydawnictwo Lekarskie PZWL, Warszawa 2015: 779-81.

7. Chang MY, Pineles SL. Pediatric Optic Neuritis. Semin Pediatr Neurol. 2017; 24(2): 122-8. https://www.sciencedirect.com/science/article/ abs/pii/S1071909117300645?via\%3Dihub (access: 17.05.2021).

8. Brenton JN. Acute Disseminated Encephalomyelitis. Medscape 2018. https://emedicine.medscape.com/article/1147044-overview.

9. Alper G. Acute disseminated encephalomyelitis. J Child Neurol. 2012; 27(11): 1408-25. http://doi.org/10.1177/0883073812455104. Epub 2012.

10. Nishiyama M, Nagase H, Tomioka K et al. Clinical time course of pediatric acute disseminated encephalomyelitis. Brain Dev. 2019; 41(6): 531-7. http://doi.org/10.1016/j.braindev.2019.02.011. Epub 2019.

Authors' contributions:

Aleksandra Świerczyńska: 50\%; Małgorzata Woś: 50\%.

Conflict of interest:

None.

Financial support:

None.

Ethics:

The content presented in the article complies with the principles of the Helsinki

Declaration, EU directives and harmonized requirements for biomedical journals. 